\title{
Cyclic Cocycles from Graded KMS Functionals
}

\author{
Daniel Kastler \\ Centre de Physique Théorique, Sect. 2, C.N.R.S., Luminy-Case 907, F-13288 Marseille Cedex 9, \\ France
}

\begin{abstract}
Each "graded KMS functional" of a $Z / 2$-graded $C^{*}$-algebra with respect to a "supersymmetric" one-parameter automorphism group gives rise to a cyclic cocycle.
\end{abstract}

In order to match algebras of primary mathematical interest for which there are no $p$-summable Fredholm modules, A. Connes introduced the wider notion of $\theta$-summable Fredholm module [1], which also encompasses the Dirac operator on loop space rigorously constructed by A. Jaffe and collaborators [2] - and subsequently developed the corresponding generalizations of cyclic cohomology and of the Chern character [3]. For constructing the latter, Connes had to resort to a "formal square root" (Ref. [3], p. 20), so to speak enforcing supersymmetry, and thus leading to conjecture a deep relationship between cyclic cohomology, supersymmetry, and the modular theory of Von Neumann algebras [4]. On the other hand A. Jaffe, A. Lesniewski and K. Osterwalder were led by the investigation of supersymmetric field theoretical models [2] to propose (under a different name) an interesting alternative construction of the Chern character of a $\theta$-summable Fredholm module [5] (cf. [9]).

The purpose of the present note is two-fold: first, using a $Z / 2$-graded version of cyclic cohomology [6,7], we enrich the (slightly adapted) Jaffe et al. (overall even) cocycle by a second component (odd both for the degree-of-form and the intrinsic grading) ${ }^{1}$. Second, we point out, as a first step towards the program [4], that the Jaffe et al. construction may be reinterpreted to pertain to "graded-KMS functionals" with respect to one-parameter automorphism groups "supersymmetric" in that they possess infinitesimal generators "with a square root." Under this aspect, [5] appears as describing the cocycle attached to the "superextension" of KMS-states of a type-I flavour. We defer to a later publication the discussion of more general cases.

\footnotetext{
${ }^{1}$ We in fact also treat the overall odd case (cf. 9 below)
} 
1. Definition. Let $A=A^{0}+A^{1}$ be a $Z / 2$-graded $C^{*}$-algebra (i.e. $A^{0}$ and $A^{1}$ are closed linear spaces with $A^{i} A^{j}\left(A^{i+j} \bmod 2\right)^{2}$ possessing a unit $\mathbb{1}$. A continuous oneparameter automorphism group of $A$ is called supersymmetric whenever

(i) $\alpha$ preserves the $Z / 2$ grading:

$$
\alpha_{t}\left(A^{i}\right) \subset A^{i}, \quad i=1,2, \quad t \in \mathbb{R},
$$

(ii) the infinitesimal generator of $\alpha$ :

$$
D=\left.\frac{d}{d t}\right|_{t=0} \alpha_{t}
$$

is the square of an odd derivation $\delta$ of $A$, i.e. one has on the domain $\mathscr{D}_{\delta}$ of $\delta$ (contained in the domain $\mathscr{D}_{D}$ of $D$ ):

$$
\begin{gathered}
D=\delta^{2}, \\
\delta(a b)=(\delta a) b+(-1)^{\partial a} a \delta b, \quad a, b \in \mathscr{D}_{\delta} \cap A^{0} \cap A^{1},
\end{gathered}
$$

[note that $(1,2),(1,3)$, and $(1,4)$ hold on the *-subalgebra $A_{\infty}$ of infinitely differentiable $(=$ smooth) elements of $A]$.

2. Definition. With $(\alpha, \delta)$ a supersymmetric one-parameter automorphism group of the $Z / 2$-graded $C^{*}$-algebra $A=A^{0}+A^{1}$, and with $t \in R$, a (bounded) linear form $\varphi$ of $A$ is called graded $t$-KMS whenever one has ${ }^{3}$

$$
\varphi(b a)=(-1)^{\partial a \hat{o} b} \varphi\left(a \alpha_{i t}(b)\right), \quad a, b \in A_{\infty} \cap A^{0} \cap A^{1},
$$

and

$$
\left.\varphi \circ \alpha_{t}=\varphi, \quad t \in R \quad \text { (hence } \varphi \circ \delta=0\right) .
$$

With these definitions one has

3. Theorem. Given a $Z / 2$-graded $C^{*}$-algebra $A=A^{0}+A^{1}$, a supersymmetric oneparameter automorphism group $(\alpha, \delta)$ of $A$ in the sense [1], and an (even $\left.{ }^{4}\right)$ graded $t$-KMS form $\varphi$ of $A$ in the sense [2], setting, for $a_{0}, a_{1}, \ldots, a_{n} \in A$,

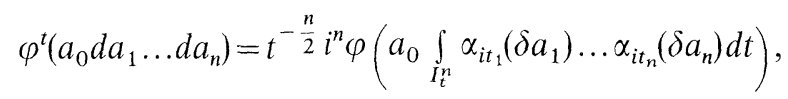

where

$$
I_{t}^{n}=\left\{t \in\left(t_{1}, \ldots, t_{n}\right) ; 0 \leqq t_{1} \leqq \ldots \leqq t_{n} \leqq t\right\}
$$

yields a cyclic cocycle of $A$ in the sense that one has

$$
\varphi^{t}(\beta \varepsilon+\mathbb{B})=0
$$

\footnotetext{
${ }^{2}$ We shall denote by $\partial a$ the grade of $a \in A^{0} \cup A^{1}$, and by $\theta$ the grading automorphism of $A$ (for $a \in A^{0}, \partial a=0$ and $\theta a=a$; for $a \in A^{1}, \partial a=1$ and $\theta a=-a$ )

${ }^{3}$ Condition (6) is not independent of (5). Note that in restriction to $A^{0}, \varphi$ is $t$-KMS in the usual sense

${ }^{4}$ Even in the sense that $\varphi$ vanishes on $A^{1}$ (could be left out, cf. 9)
} 
where ${ }^{5} \beta \varepsilon=\beta^{\prime} \varepsilon-\alpha \varepsilon$ with, for $a_{0}, a_{1}, \ldots, a_{n+1} \in A^{0} \cup A^{1}$,

$$
\begin{aligned}
\beta^{\prime} \varepsilon\left(a_{0} d a_{1} \ldots d a_{n+1}\right)= & (-1)^{\partial a_{0}} a_{0} a_{1} d a_{1} \ldots d a_{n+1} \\
& +\sum_{j=1}^{n}(-1)^{j+\sum_{k=0}^{j} \partial a_{k}} a_{0} d a_{1} \ldots d\left(a_{j} a_{j+1}\right) \ldots d a_{n+1}, \\
\alpha \varepsilon\left(a_{0} d a_{1} \ldots d a_{n+1}\right)= & (-1)^{\left(1+\hat{o} a_{n+1}\right)\left(n+\sum_{k=0}^{n} \hat{\partial} a_{k}\right)} a_{n+1} a_{0} d a_{1} \ldots d a_{n},
\end{aligned}
$$

and $\mathbb{B}=\mathbb{B}_{0} A$ with

$$
\mathbb{B}_{0}\left(a_{0} d a_{1} \ldots d a_{n}\right)=\mathbb{1} d a_{0} d a_{1} \ldots d a_{n}+(1)^{n+\sum_{k=0}^{n} \delta a_{h}} a_{0} d a_{1} \ldots d a_{n} d \mathbb{1},
$$

and $A=\sum_{k=0}^{n} \lambda^{n}$ on $\Omega^{n}$, where

$$
\lambda\left(a_{0} d a_{1} \ldots d a_{n}\right)=(-1)^{\left(1+\partial a_{n}\right)\left(n+{ }_{k=0}^{n-1} \partial a_{k}\right)} a_{n} d a_{0} d a_{1} \ldots d a_{n-1} .
$$

In fact one has

$$
\begin{aligned}
\varphi^{t} \circ \beta \varepsilon\left(a_{0} d a_{1} \ldots d a_{n}\right) & =t^{\frac{n-1}{2}} i^{n-1} \varphi\left(\delta a_{0} \int_{I^{n}} \alpha_{i t_{1}}\left(\delta a_{1}\right) \ldots \alpha_{i t_{n}}\left(\delta a_{n}\right) d t\right) \\
& =-\varphi^{t} \circ \mathbb{B}\left(a_{0} d a_{1} \ldots d a_{n}\right),
\end{aligned}
$$

The proof follows from a sequence of lemmas.

4. Lemma. With $u_{i}, i=1, \ldots, n$ differentiable functions: $\mathbb{R} \rightarrow A$, setting $f_{(1)}^{t}=\mathbb{1}$ and

$$
f_{(n)}^{t}\left(u_{1}, \ldots, u_{n}\right)=\int_{I_{t}^{n}} u_{1}\left(t_{1}\right) \ldots u_{n}\left(t_{n}\right) d t, \quad t \in \mathbb{R}
$$

we have that, with $\dot{u}_{i}=\frac{d}{d t} u_{i}, i=1, \ldots, n$, for $1<k<n, n=1,2, \ldots$ :

$$
\begin{aligned}
& f_{(n)}^{t}\left(\dot{u}_{1}, u_{2}, \ldots, u_{n}\right)=f_{(n-1)}^{t}\left(u_{1} u_{2}, u_{3}, \ldots, u_{n}\right)-u_{1}(0) f_{(n-1)}^{t}\left(u_{2}, \ldots, u_{n}\right) \\
& f_{(n)}^{t}\left(u_{1}, \ldots, \dot{u}_{k}, \ldots, u_{n}\right)=f_{(n-1)}^{t}\left(u_{1}, \ldots, u_{k} u_{k+1}, \ldots, u_{n}\right)-f_{(n-1)}^{t}\left(u_{1}, \ldots, u_{k-1} u_{k}, \ldots, u_{n}\right) \\
& f_{(n)}^{t}\left(u_{1}, \ldots, u_{n-1}, \dot{u}_{n}\right)=f_{(n-1)}^{t}\left(u_{1}, \ldots, u_{n-1}\right) u_{n}(t)-f_{(n-1)}^{t}\left(u_{1}, \ldots, u_{n-2}, u_{n-1} u_{n}\right)
\end{aligned}
$$

and, with $\mathbb{1}$ the constant unit function,

$$
\sum_{k=1}^{n-1} f_{(n+1)}^{t}\left(u_{1}, \ldots, u_{k}, \mathbb{1}, u_{k+1}, \ldots, u_{n}\right)=t f_{(n)}^{t}\left(u_{1}, \ldots, u_{n}\right) .
$$

Proof. Equation (16) follows straightforwardly from (15); and (17) by termwise adding the relations obtained by making $\dot{u}_{k}=\mathbb{1}\left(u_{k}(t)=t \mathbb{1}\right)$ in $(16)$ for $k=1, \ldots, n$.

5. Lemma. Setting, for $a_{0}, a_{1}, \ldots, a_{n} \in A^{0} \cup A^{1}$,

$$
\Psi^{t}\left(a_{0} d a_{1} \ldots d a_{n}\right)=a_{0} f_{(n)}^{t}\left(\delta \underline{a}_{1}, \ldots, \delta \underline{a}_{n}\right),
$$

\footnotetext{
${ }^{5}$ We have used the definition of the Hochschild boundary $\beta \varepsilon$ and the operator $\lambda$ of $Z / 2$-graded cyclic cohomology as formulated within the differential envelope $\Omega=\oplus_{n} \Omega^{n}$ [6]. For the formulation in terms of multilinear forms, see 6 below
} 
where $\underline{a}_{n}$ denotes the function $t \rightarrow \alpha_{i t}\left(a_{n}\right), k=1, \ldots, n,\left(\right.$ so that $\left.\varphi^{t}=t^{-\frac{n}{2}} i^{n} \varphi \Psi^{t}, c f .(7)\right)$ we have, for ${ }^{6} \omega \in \Omega^{0} \cup \Omega^{1}, a \in A^{0} \cup A^{1}, b \in A$ :

$$
\Psi^{t}\left(\beta^{\prime} \varepsilon(a d \omega d b)\right)-(-1)^{\hat{o}(a d \omega)} \Psi^{t}(a d \omega) \Psi^{t}\left(\alpha_{i t}(b)\right)=\delta \Psi^{t}(a d \omega d b)-\delta a \Psi^{t}(\mathbb{1} \omega d b),
$$

where $\beta^{\prime} \varepsilon$ is the operator $(10)$.

Proof. For $a_{0}, a_{1}, \ldots, a_{n} \in A^{0} \cup A^{1}$ we have, using the derivation rule (4), and relations (3) and (16),

$$
\begin{aligned}
& -(1)^{\partial a_{0}} a_{0} \delta\left\{f_{(n)}^{t}\left(\delta \underline{a}_{1}, \ldots, \delta \underline{a}_{n}\right)\right\} \\
& =(-1)^{\partial a_{0}} a_{0} a_{1} f_{(n-1)}^{t}\left(\delta \underline{a}_{2}, \ldots, \delta \underline{a}_{n}\right) \\
& +\sum_{j=1}^{n-1}(-1)^{j+\sum_{k=0}^{j} \partial a_{k}} a_{0} f_{(n-1)}^{t}\left(\delta \underline{a}_{1}, \ldots, \delta\left(\underline{a}_{j} \underline{a}_{j+1}\right), \ldots, \delta \underline{a}_{n}\right)
\end{aligned}
$$

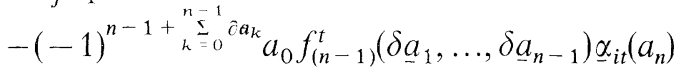

$$
\begin{aligned}
& =-\delta\left\{a_{0} f_{(n)}^{t}\left(\delta \underline{a}_{1}, \ldots, \delta \underline{a}_{n}\right)\right\}+\delta a_{0} f_{(n)}^{t}\left(\delta \underline{a}_{1}, \ldots, \delta \underline{a}_{n}\right),
\end{aligned}
$$

yielding (19) for $a_{0}=a, a_{n}=b, \omega=d a_{1}, \ldots, d a_{n-1}$.

Equating the values for both sides of (19) of a graded $t$-KMS linear form $\varphi$ of $A$ then yields the first equations (14), since $^{7}$

$$
\begin{aligned}
(-1)^{\partial(a d \omega)} \varphi\left\{\Psi^{t}(a d \omega) \Psi^{t}\left(\alpha_{i t}(b)\right)\right\} & =(-1)^{\hat{\partial}(a d \omega)(\partial b+1)} \varphi\left\{\Psi^{t}(\kappa) \Psi^{t}(a d \omega)\right\} \\
& =\varphi\left\{\Psi^{t}(\alpha(a d \omega d \kappa))\right\}
\end{aligned}
$$

For the proof of the second equation (14) we need

6. Lemma. Let $\varphi$ be an even graded t-KMS linear form of $A$, and set, for $a_{0}$, $a_{1}, \ldots, a_{n} \in A$,

$$
F_{(n)}^{t}\left(a_{0}, a_{1}, \ldots, a_{n}\right)=\varphi\left(a_{0} f_{(n)}^{t}\left(\underline{a}_{1}, \ldots, \underline{a}_{n}\right) .\right.
$$

We have the properties

$$
F_{(n)}^{t}\left(a_{n} a_{0}, a_{1}, \ldots, a_{n-1}\right)=(-1)^{\partial a_{n}} F_{(n)}^{t}\left(a_{0}, a_{1}, \ldots, a_{n}\right), \quad a_{n} \in A^{0} \cup A^{1},
$$

and

$$
\sum_{k=0}^{n} F_{(n+1)}^{t}\left(a_{0}, \ldots, a_{k}, \mathbb{1}, \ldots, a_{n}\right)=t F_{(n)}^{t}\left(a_{0}, a_{1}, \ldots, a_{n}\right)
$$

Proof. Using (5) and (6) we have

$$
\begin{aligned}
& F_{(n)}^{t}\left(a_{0}, a_{1}, \ldots, a_{n}\right) \\
& \quad=\int_{t \in I_{t}^{n}} \varphi\left\{a_{0} \alpha_{i t_{1}}\left(a_{1}\right) \ldots \alpha_{i t_{n}}\left(a_{n}\right)\right\} d t \\
& \quad=(-1)^{\hat{\partial} a_{n}{ }_{k=0}^{n-1} \hat{\partial} a_{k}} \int_{t \in I_{t}^{n}} \varphi\left\{a_{n} \alpha_{i\left(t-t_{n}\right)}\left(a_{0}\right) \alpha_{i\left(t+t_{n}-t_{1}\right)}\left(a_{1}\right) \ldots \alpha_{i\left(t+t_{n-1}-t_{n}\right)}\left(a_{n-1}\right)\right\} d t,
\end{aligned}
$$

${ }^{6} \Omega^{0}$ and $\Omega^{1}$ are the even, respectively odd parts of the differential envelope $\Omega$ for its total grading (sum of the $n$-grading and the intrinsic grading). The total grade of $\omega \in \Omega^{0} \cup \Omega^{1}$ is denoted $\partial \omega$ ${ }^{7}$ Note that the first equation (14) holds for all graded $t$-KMS linear forms of $A$, irrespective of parity 
however, with $s=\left(s_{1}, \ldots, s_{n}\right), s_{1}=t-t_{n}, s_{2}=t-t_{n}+t_{1}, \ldots, s_{n}=t-t_{n}+t_{n-1}$, one has $t \in I_{t}^{n}$ iff $s \in I_{t}^{n}$; and $\varphi$ is even, i.e. vanishes unless $\sum_{k=0}^{n} \partial a_{n}=0$ : this proves (23). As for (24), it immediately follows from (22) and (17).

We now check the second equation (14): rewriting definition (7) as

$$
\varphi^{t}\left(a_{0} d a_{1} \ldots d a_{n}\right)=t^{-\frac{n}{2}} i^{n} F_{(n)}^{t}\left(a_{0}, \delta a_{1}, \ldots, \delta a_{n}\right),
$$

we have from (12), since $\delta \mathbb{1}=0$, and using (23),

$$
\varphi^{t} \circ \mathbb{B}_{0}\left(a_{0} d a_{1} \ldots d a_{n}\right)=t^{-\frac{n+1}{2}} i^{n+1} F_{(n+1)}^{t}\left(\delta a_{0}, \delta a_{1}, \ldots, \delta a_{n}, \mathbb{1}\right),
$$

hence, since $\varphi$, and thus $F_{(n+1)}^{t}$, is even

$$
\varphi^{t} \circ \mathbb{B}_{0} \lambda^{k}\left(a_{0} d a_{1} \ldots d a_{n}\right)=t^{-\frac{n+1}{2}} i^{n+1} F_{(n+1)}^{t}\left(\delta a_{0}, \ldots, \delta a_{n-k}, \mathbb{1}, \ldots, \delta a_{n}\right),
$$

whence our result, by termwise addition.

7. Remark. As explained in $[6]$ Remark $[3,5]$, the following regauging of $\varphi^{t}$ :

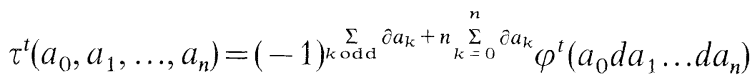

will produce the cocycle condition $(b+B) \tau^{t}=0$, where

$$
\begin{aligned}
\left(b \tau^{t}\right)\left(a_{0}, a_{1}, \ldots, a_{n}\right)= & \sum_{j=0}^{n-1}(-1)^{j} \tau^{t}\left(a_{0}, \ldots, a_{j} a_{j+1}, \ldots, a_{n}\right) \\
& -(-1)^{n-1+\partial a_{n} \sum_{k=0}^{n-1} \partial a_{k}} \tau^{t}\left(a_{n} a_{0}, a_{1}, \ldots, a_{n-1}\right),
\end{aligned}
$$

and $B=A B_{0}$ with

$$
\left(B_{0} \tau^{t}\right)\left(a_{0}, a_{1}, \ldots, a_{n}\right)=\tau^{t}\left(\mathbb{1}, a_{0}, \ldots, a_{n}\right)
$$

and $A=\sum_{k=0}^{n} \lambda^{k}$, where

$$
\left(\lambda \tau^{t}\right)\left(a_{0}, \ldots, a_{n}\right)=(-1)^{n+\partial a_{n} \sum_{k=0}^{n-1} \hat{c} a_{k}} \tau^{t}\left(a_{n}, a_{0}, a_{1}, \ldots, a_{n-1}\right) .
$$

8. Remark. In a quantum field theory situation we know from [8] that any extremal invariant $\beta$-KMS (temperature) state of the bosonic part $A^{0}$ extends uniquely to a state $\varphi$ of $A$ invariant for $\alpha(\mathbb{R})$ and $\theta$ and such that

$$
\varphi(b a)=\varphi\left\{a\left(\alpha_{i \beta} \circ \gamma\right)(b)\right\}, \quad a, b \in A
$$

with $\gamma=\mathrm{id}$ but, for $\varphi$ odd, (32) is a reformulation of (5).

9. Remark. Theorem 3 holds as well for odd (graded=ordinary) $t$-KMS forms. Indeed, as one checks easily, for $\varphi$ odd relation (23) holds without the sign factor right hand side, whilst (26) and (27) hold as they stand.

\section{References}

1. Connes, A.: Compact metric spaces, Fredholm modules and hyperfiniteness. IHES preprint

2. Jaffe, A., Lesniewski, A., Weitsman, J.: Index of a family of Dirac operators on loop space. Commun. Math. Phys. 112, 75 (1987) 
3. Connes, A.: Entire cyclic cohomology of Banach algebras and characters of $\theta$-summable Fredholm modules. IHES preprint $\mathrm{M} / 87 / 46$

4. Connes, A.: Private communication

5. Jaffe, A., Lesniewski, A., Osterwalder, K.: Quantum K-theory. I. The Chern character. Commun. Math. Phys. 118, 1-14 (1988)

6. Kastler, D.: Cyclic cohomology within the differential envelope. An introduction to A. Connes, non commutative differential geometry. Paris: Hermann 1988

7. Kastler, D.: Entire cyclic cohomology of $Z / 2$-graded Banach algebras. Marseille preprint CPT $88 / \mathrm{I} 2128$

8. Araki, H., Haag, R., Kastler, D., Takesaki, M.: Extension of KMS states and chemical potential. Commun. Math. Phys. 35, 97 (1977)

9. Kastler, D.: Introduction to entire cyclic cohomology of $\mathbb{Z} / 2$-graded Banach algebras. Franco-German Meeting. Ph. Blanchard Editor. In print Kluwer

Communicated by A. Connes

Received September 23, 1988

Note added in proof. Theorem 3 suggests the following questions:

(i) In which situations is the entire cohomology class independant of temperature (as found in [5])? If this prevails in physics, to which extent is the construction of relativistic supersymmetric field theories tantamount to computing the entire cyclic cohomology of a universal algebra (array of local type IIIs with intermediate type Is)?

(ii) Are the KMS-states the adequate generalization of clliptic operators to the noncommutative (possibly type III) frame? 